ENCYCIOPEDDE Encyclopédie berbère

BERBERE $\quad \mathbf{3 3} \mid \mathbf{2 0 1 2}$

33| N - Nektiberes

\title{
Navigation : Antiquité
}

\section{Christine Hamdoune}

\section{OpenEdition}

Journals

Édition électronique

URL : https://journals.openedition.org/encyclopedieberbere/2689

DOI : 10.4000/encyclopedieberbere.2689

ISSN : 2262-7197

\section{Éditeur}

Peeters Publishers

\section{Édition imprimée}

Date de publication : 4 octobre 2012

Pagination : $5299-5310$

ISBN : 978-90-429-2640-0

ISSN : 1015-7344

\section{Référence électronique}

Christine Hamdoune, "Navigation : Antiquité », Encyclopédie berbère [En ligne], 33 | 2012, document N20, mis en ligne le 23 novembre 2020, consulté le 17 février 2022. URL : http://

journals.openedition.org/encyclopedieberbere/2689; DOI : https://doi.org/10.4000/ encyclopedieberbere.2689

Ce document a été généré automatiquement le 17 février 2022.

(c) Tous droits réservés 


\title{
Navigation : Antiquité
}

\author{
Christine Hamdoune
}

1 Les Africains, malgré l'étendue des façades maritimes sur la Méditerranée et l'Atlantique, s'intéressèrent assez tard à la navigation ; les relations de l'Afrique et de la mer sont le résultat d'une longue histoire qui commence avec l'arrivée des Phéniciens dans le bassin occidental de la Méditerranée, un espace plus tardivement maîtrisé par les navigateurs, comme le montrent bien ses divisions en différents secteurs beaucoup plus vastes que dans la partie orientale, d'après le schéma défini par les Grecs et repris par les Romains (Pline); ce décalage est également illustré par les descriptions de l'Odyssée, beaucoup plus discrètes sur la Méditerranée occidentale que sur l'Égée. Certes il y a eu des premières navigations dès le néolithique et, à l'âge du bronze récent (XIV ${ }^{\mathrm{e}}$-XIII siècles a.C.), des liens commerciaux existent, entre l'Orient, l'Égée et le reste de la Méditerranée, que n'ont pas interrompus la disparition du monde mycénien et les bouleversements dans le levant liés à l'invasion des peuples de la mer. Mais en l'état actuel des connaissances (qui reposent sur la présence d'objets orientalisants en contexte archéologique), c'est au IX siècle a.C. que les habitants de Phénicie, les Tyriens surtout, ont commencé à être durablement présents en Méditerranée occidentale. Ils sont les premiers à s'aventurer le long des côtes africaines, à y installer, pour leur commerce, des comptoirs sur les îles, comme l'îlot tabulaire de Rachgoun (Mersa Madakh en face de l'embouchure de la Tafna, en Oranie), et sur les estuaires, profitant des rares mouillages le long d'une côte souvent inhospitalière (Salluste, J. 17, 5 , « la mer y est mauvaise, sans mouillage »), soit par la présence de hauts fonds réputés dangereux, comme les Syrtes, soit à cause de l'allure escarpée des rivages, comme à l'ouest du Cap Blanc, et entre Tabarka et Hippone, ou encore, comme au sud du Cap Bon, à cause de l'allure des vents et des courants. On retrouve le caractère aléatoire des mouillages dans les périples, tel celui du Pseudo-Scylax, au milieu du IV siècle, mais plus encore dans le dernier d'entre eux, le stadiasmus maris magni, rédigé au $\mathrm{III}^{\mathrm{e}}$ siècle p.C., qui, à partir d'une compilation de documents d'époques différentes, offre une documentation remarquable pour connaître aussi bien les toponymes côtiers que leurs distances et la qualité des mouillages. Les Phéniciens fondent Utique et franchissent les Colonnes d'Hercule pour s'établir à Gadès en Espagne et à Lixus le long de la côte atlantique du Maroc, atteignent l'îlot de Mogador au VII siècle. Un peu plus tard, vers 
600 , ils réussissent à faire le tour de l'Afrique, pour le compte du pharaon Nechao (Hérodote, IV, 42-43). C'est dans ce contexte que s'est développé le plus important de leur comptoir, Carthage, fondé à l'extrême fin du IX siècle, qui va progressivement supplanter les Phéniciens, sans qu'il soit toujours possible de déterminer exactement, surtout dans la région du détroit de Gibraltar, ce qui relève de Carthage indépendamment de sa cité mère. L'expansion carthaginoise s'affirme à partir du $\mathrm{VI}^{\mathrm{e}}$ siècle, attestée par le premier traité signé en 509 avec Rome qui interdit aux navires romains de dépasser le cap Bon, truffé de postes de garde pour protéger le commerce des Carthaginois avec les cités du Sahel et des Syrtes. Dès cette date, les Carthaginois considèrent l'Afrique comme leur propre domaine (Polybe, III, 2), un domaine sur lequel ils se concentrent de plus en plus, après leurs échecs, d'abord contre les Grecs de Sicile à Himère (480 a.C.), puis contre les Romains dans les première et seconde guerres puniques qui leur font perdre la Sicile, la Sardaigne et l'Espagne. Cette emprise de Carthage sur le littoral africain se traduit par la multiplication d'escales depuis l'autel des Philènes à l'est (Lepcis magna et Sabratha), le long des côtes du Sahel (Hadrumète), dans le cap Bon (Clupea), puis au nord-ouest (Bizerte, Tabarka) et tout le long des côtes algériennes. Si les conditions de la navigation imposent parfois de longues distances entre deux mouillages, comme entre Tabarka et Hippone, on constate, à partir de la petite Kabylie, une succession de sites dont les noms sont d'origine punique (Rusazus, Rusuccurru, Rusguniae) et une dissémination du matériel archéologique en de nombreux autres points jusqu'au cap Spartel.

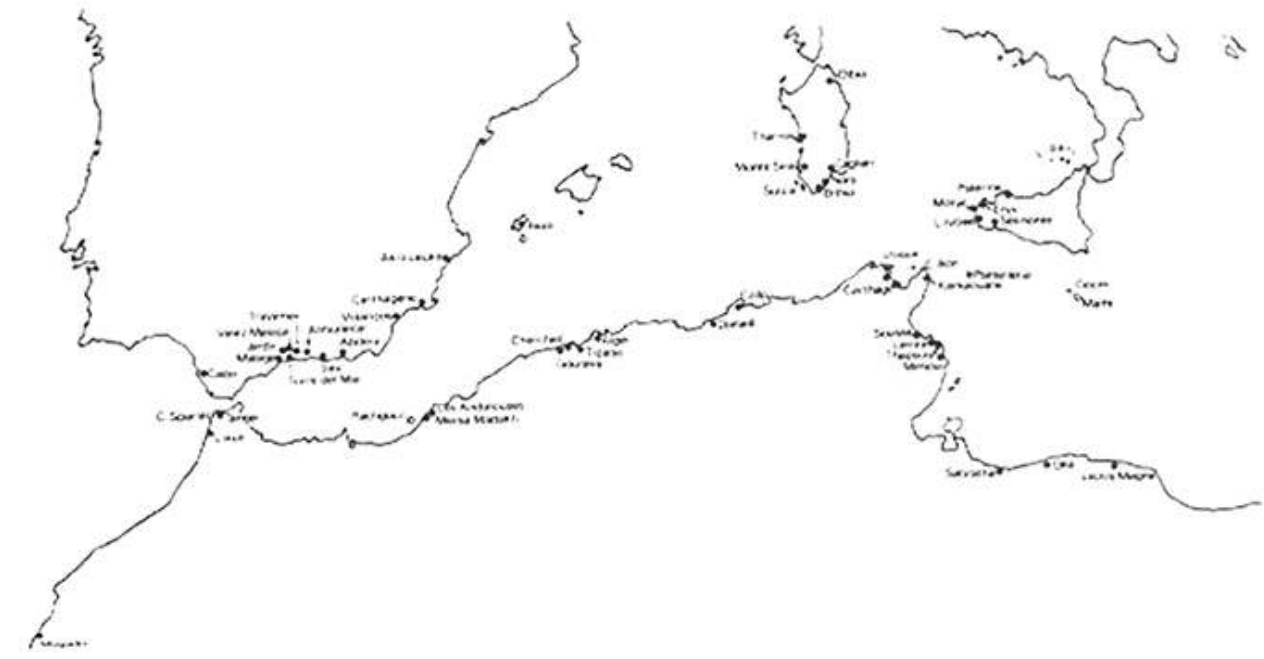

CARTE des escales Puniques d'APRÈs S. LANCEL, CARTHAge, P. 98

2 Il faut cependant nuancer la notion d'échelles puniques élaborée par Cintas qui, se fondant sur une capacité de navigation côtière et uniquement diurne, avait proposé de voir, dans chacun de ces mouillages, une escale entièrement punique, obligatoire tous les 30 à $40 \mathrm{~km}$. En réalité, les établissements côtiers sont beaucoup plus diversifiés et correspondent souvent à des communautés libyques auxquelles les Carthaginois vendaient et achetaient, comme le montre bien l'exemple de Tipasa, où se mêlent influences puniques et maintien de traditions autochtones, et celui de Césarée, où l'on note, comme à Rachgoun, un glissement depuis l'îlot, occupé très anciennement, vers la terre ferme et les vestiges de l'établissement autochtone. Ces relations avec les populations locales et la façon dont elles pouvaient s'organiser à très haute époque sont attestées par le témoignage d'Hérodote sur le troc entre marchands carthaginois et 
indigènes le long du littoral atlantique (IV, 196), mais aussi par la gravure rupestre d'un navire dans la paroi de la berge de l'oued Draa au sud du Maroc.

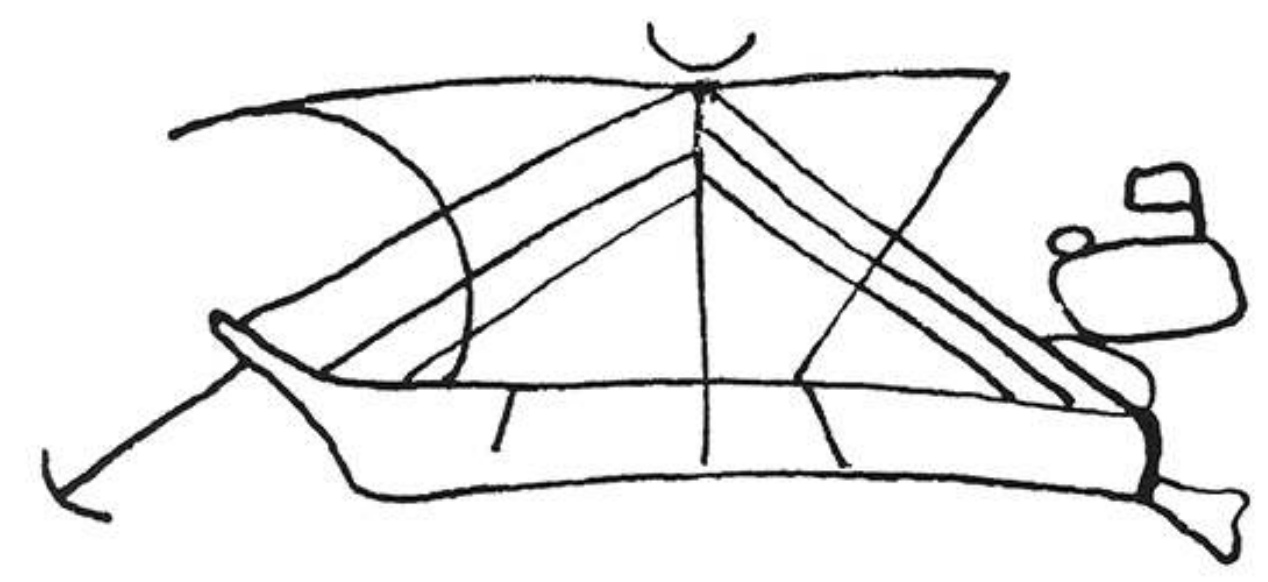

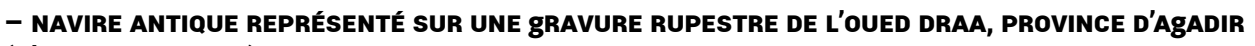
(D'APRÈS R. MAUNY).

3 En effet, comme les Phéniciens, les Carthaginois ont dépassé les Colonnes d'Hercule et entretiennent la tradition des grands périples dont le plus célèbre est celui d'Hannon, un récit de voyage connu par une version manuscrite d'un texte grec qui était affiché dans le temple de Baal Hammon à Carthage : le roi Hannon, parti peut-être de Gadès avec 60 vaisseaux à 50 rames (pentécontores) portant 30000 hommes et femmes, navigua le long des côtes atlantiques du Maroc pour fonder des villes. Si le récit est le plus souvent défiguré, surtout dans la description des régions méridionales, par le goût hellénistique pour les mirabilia, la réalité d'un voyage entre le $\mathrm{VI}^{\mathrm{e}}$ et le $\mathrm{IV}^{\mathrm{e}}$ siècle est assurée, au moins jusqu'à Lixus, voire jusqu'à Mogador où il faudrait peut-être replacer Cernè. On en retiendra la capacité des Carthaginois à tirer parti des expériences acquises dans la connaissance des phénomènes naturels (vents, courants, allure des côtes, observation des astres, écueils, hauts fonds, caps, amers et distances entre lieux de mouillage) indispensables à la navigation dans l'antiquité qui, en l'absence d'instrument de mesure, se faisait à l'estime ; mais aussi la complexité des échanges qui pouvaient, dans la partie la plus occidentale de l'espace méditerranéen, s'organiser moins à partir de Carthage que des établissements ibériques avec le transport de marchandises locales et grecques, comme l'illustre le site de Kouass au Maroc, voire étrusques. Ces données montrent qu'il faut en fait envisager le problème de la navigation selon deux échelles différentes, celle des relations de l'Afrique avec le reste de la Méditerranée occidentale et celle plus restreinte du rôle de la navigation dans les échanges de redistribution locale. Dès l'époque carthaginoise, devait donc exister le schéma d'organisation du commerce maritime, plus affirmé encore à l'époque romaine, décrit par P. Pomey qui distingue des ports principaux, points de départ et d'aboutissement de navires à la cargaison homogène et gros porteurs, aptes à une navigation au long cours soit en longeant les côtes soit en haute mer, et où s'opèrent le déchargement et le stockage des marchandises; de là, sur des embarcations plus petites, des cargaisons hétérogènes étaient acheminées, par cabotage, vers les ports secondaires situés dans la zone d'influence du port principal. A partir de là, la redistribution s'opérait vers l'intérieur, par la voie fluviale quand c'était possible (Pline par exemple insiste sur la navigabilité de certains oueds, comme le Sebou au Maroc) ou 
par route. Ces deux échelles sont particulièrement sensibles le long de la côte algérienne comme l'a récemment montré J.-P. Laporte (2008) :

A
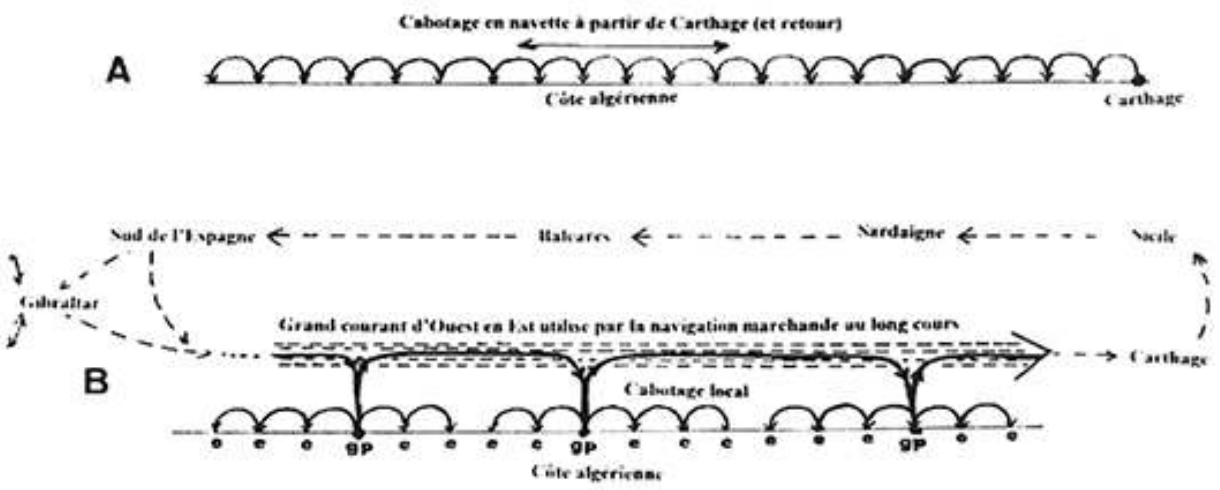

DEUX MOdÈles de NAVIgATION LE LONg DES CôteS ALgÉRIENNES. A : MOdÈle CINTAS. B : NOUVEAU MODĖLE.

Dessins J.-P. LAPORTE.

4 La connaissance des vents dominants de force et d'orientation compatibles avec la direction souhaitée permettait d'effectuer une navigation hauturière qu'autorisaient les progrès dans la construction navale, avec l'usage du bitume et le façonnement des coques à membrures, qui rendaient les navires aptes à affronter la haute mer. L'îlot de Pantelleria, au large du cap Bon, joue, dans ces routes, un rôle de relais incontournable : c'est un amer (836 m d'altitude) au carrefour de plusieurs itinéraires, mais aussi à l'articulation de deux systèmes météorologiques constituant une zone de rupture de charge qui explique la variété des produits qu'on y rencontre et confirme l'existence des relations à grande distance. Mais cette navigation hauturière est une navigation segmentée, liée à un parcours possible durant une période déterminée car la majorité des grands itinéraires n'étaient praticables qu'en un seul sens et pendant une période précise de l'année. La route est-ouest au départ de Carthage vers l'Espagne passait sans doute par le sud de la Sardaigne et les Baléares, alors que la circulation dans l'autre sens se faisait plus près des côtes en utilisant le grand courant ouest-est. De tels voyages limitaient le nombre des escales sans minimiser le rôle du cabotage qui recouvre en fait deux réalités : une forme de navigation et une structure d'échanges.

La puissance de Carthage était donc fondée sur le négoce et le contrôle des circuits commerciaux et la marine tenait un grand rôle. Le principal navire de commerce était le gaulos, un navire ventru avec deux bancs de nage mais sans voile, déjà utilisé par les Phéniciens (voir les bas-reliefs du palais de Sennacherib à Ninive, datés du VIII ${ }^{\mathrm{e}}$ siècle). Un objet votif carthaginois en terre cuite, conservé à Paris, représente l'un de ces navires de charge : il est cependant doté d'une voile et moins large que le gaulos. Un autre type de bateau, l'hippos, plus petit et symétrique, avait un mât et un cheval comme figure de proue (Strabon, II, 3, 4 [C99-100] dit que les Gaditains l'utilisaient pour la pêche notamment jusqu'au sud de Lixus). Il fallait bien sûr protéger les intérêts des marchands et la flotte de guerre carthaginoise s'est développée au moment de la rivalité avec les Grecs de Sicile : elle est attestée par Diodore de Sicile (XI, 1,5; 20, 2 et XIII, 54, 1) pour l'expédition d'Hamilcar en 480 et par l'archéologie sous-marine avec la récupération, à Motya, près de Marsala, de deux épaves de navires de guerre puniques du III $^{\mathrm{e}}$ siècle, dotés d'un unique rang de rameurs et armés d'un éperon, qui renseignent aussi sur les techniques de construction des charpentiers. Mais les Carthaginois 
construisirent aussi des trirèmes et quinquérèmes. Ces bateaux de guerre possédaient, comme souvent dans l'Antiquité, leur propre port, distinct du port marchand. Or, pour les cinq premiers siècles de l'histoire de Carthage, on ne connaît pas les ports de la cité ; à l'origine, le port aurait été aménagé dans une large échancrure du lac de Tunis remontant vers le $\mathrm{N}$ et l'E au moins jusqu'à hauteur du tophet; les deux ports furent aménagés dans leur version finale dans la première moitié du $\mathrm{II}^{\mathrm{e}}$ siècle. Ils sont très visibles dans le paysage avec les deux lagunes qui correspondent à la description qu'en a laissée Appien d'après celle de Polybe. Les fouilles britanniques ont reconnu le port de guerre circulaire, qui a occupé un espace précédemment voué aux chantiers navals, avec une série de cales sous forme de rampes; le port marchand communiquait par un chenal avec la mer et là encore des traces de quais du II ${ }^{\mathrm{e}}$ siècle ont été retrouvées (voir EB, XII, Carthage, p. 1784-1785).

Dans cet espace ainsi dominé par Carthage, il faut attendre la fin du III ${ }^{e}$ siècle pour que les Libyens s'intéressent davantage à la mer. Jusque-là, le rôle de la navigation s'était traduit par des influences culturelles. C'est avec le roi Syphax que l'on a les premières manifestations de cette prise de conscience de l'importance de la navigation. Il installe sa capitale à l'embouchure de la Tafna, à proximité de l'îlot de Rachgoun, le plus vieil établissement phénicien sur la côte algérienne. Après la fin de la première guerre punique, il est probable que l'emprise carthaginoise sur les escales maghrébines entre le cap Bougaroun et le cap Spartel qui jalonnaient la route vers l'Espagne barcide, se fit plus étroit, formant le Metagonium. Syphax a dû être sensible à cet aspect et revendiquer la suzeraineté sur les villes côtières, comme Césarée. Le fait de recevoir, en 206, Hasdrubal et Scipion, le premier depuis Gadès, le second depuis Carthagène, reflète bien cette prise de conscience et en même temps montre le rôle de l'Espagne dans l'ouverture de royaumes jusque-là très continentaux sur la mer. Le traité de 201 signifia la disparition du Metagonium punique en tant que structure de pouvoir. De plus Massinissa s'empara au cours du $\mathrm{II}^{\mathrm{e}}$ siècle de la plus grande partie des emporia puniques de la petite Syrte et de Tripolitaine. Est-ce à dire que le royaume numide supplanta Carthage en tant que grande puissance maritime? Tel est l'avis de G. Camps (p. 196) qui considère que Massinissa possédait une flotte de guerre importante, d'après une remarque de Cicéron (Verrines, II, IV, 103), reprise par Valère Maxime, (I, I, ext. 2), relative à un acte de piraterie commis par l'un des préfets de la flotte du roi qui avait dérobé des défenses d'ivoire dans un sanctuaire de Junon à Malte. Pour Camps, Massinissa possédait suffisamment de navires pour distraire un quinquérème de ses activités normales vers Malte. D'autre part, les navires de commerce du royaume dynamisaient des échanges actifs avec l'Orient hellénistique, notamment Rhodes et Délos où le roi exportait le thuya et l'ivoire mais aussi du blé. En fait, les relations avec la Grèce étaient plus d'ordre politique et culturel et les travaux de V. Bridoux montrent que les royaumes africains, à partir du II ${ }^{\mathrm{e}}$ siècle, sont entrés dans la sphère économique dominée par Rome qui a récupéré à son profit les axes d'échange puniques et, pour certains (le Metagonium), avant même la destruction de Carthage, par l'intermédiaire de relations privilégiées depuis la Bétique dans la zone du détroit. Peut-être vaut-il mieux alors en revenir à la prudence de S. Gsell (HAAN, V- Les royaumes indigènes, 1929, $2^{\mathrm{e}}$ éd., p.151-152) qui évoque, à propos des mêmes textes, une flotte modeste destinée à réprimer les actes de piraterie, à moins qu'elle ne s'y adonnât elle-même. En effet, et de plus en plus nettement, d'après le témoignage de la céramique campanienne (voir l'exemple de Banasa et de Kouass récemment), l'essentiel du commerce est aux mains des Italiens qui intègrent l'Afrique dans leurs circuits commerciaux et assurent les 
exportations de blé notamment vers Rome. Cette tendance s'accentue tout au long du siècle, comme le montre la présence des negotiatores italiens à Vaga au début de la guerre de Jugurtha. L'investissement des Africains dans la vie maritime est faible et l'on peut en trouver une attestation dans les thèmes du monnayage des cités autonomes du $\mathrm{I}^{\mathrm{er}}$ siècle: les représentations marines sont rares, à l'exception de celle des deux poissons à Lixus (mais que l'on doit mettre en relation avec les activités de la fabrication du garum) et de celle du dauphin à Césarée. La même tendance se retrouve à l'ouest dans le royaume maurétanien ; l'histoire d'Eudoxe de Cyzique (Strabon, II, 3, 4) au début du $\mathrm{I}^{\text {er }}$ siècle le montre bien : si le roi Bocchus lui fait bon accueil au départ, il retire vite son appui financier pour la préparation de l'expédition maritime projetée le long des côtes africaines. Au moment de la guerre d'Afrique, Varus recrute des marins gétules à Utique $(B A, 62,1)$, des sujets de Juba $\mathrm{I}^{\mathrm{er}}$, mais cette notation ne donne aucun indice sur l'existence d'une flotte importante dans le royaume numide, surtout quand on la compare aux nombreuses allusions aux diverses troupes de cavaliers et fantassins et aux éléphants fournis par le roi aux pompéiens. Au même moment, le roi maurétanien, Bocchus, utilise les services de Sittius, un ancien négociant campanien, exilé après la conjuration de Catilina, pour rétablir l'ordre sur mer dans son royaume et participer à la guerre contre les pompéiens. C'est avec le règne de Juba II, que l'on peut véritablement mesurer l'ouverture du royaume maurétanien vers la mer. Le roi dote sa capitale d'un port remarquable précédé d'un phare dans l'îlot (Leveau, Césarée, p. 47-51) : il comprenait un port extérieur, fermé par deux jetées et communiquant par un goulet étroit avec un port intérieur entouré de portiques et de magazins. Le roi possède une flotte capable d'aller aux Canaries (Pline, VI, 203) et de rapporter la pourpre de Mogador. Cette flotte royale peut être à l'origine du maintien d'une escadre de la marine romaine plus tard.

7 L'intérêt du roi maurétanien pour la navigation reflète l'importance croissante des relations maritimes dans un espace devenu le mare nostrum, rarement troublé dans sa partie occidentale par des actes de piraterie isolés. La piraterie a, en effet, été un mal endémique jusqu'au début de l'empire, comme le montre l'épave de Mahdia, découverte en 1907 à $39 \mathrm{~m}$. de profondeur : il s'agit d'un grand navire d'environ $30 \mathrm{~m}$ de long, lourdement chargé $(250 \mathrm{t}$.) par une cargaison de sculptures de marbre et de bronze et d'éléments d'architecture (70 colonnes avec socle et chapiteau). Le bateau en provenance d'Athènes pillée par Sylla, faisait route vers Rome, mais avait été détourné de sa destination. Un réexamen récent du matériel a montré que certaines pièces de bronze faisaient partie d'une catapulte qui armait le vaisseau pour sa défense contre des pirates. À partir du règne d'Auguste, les actes de piraterie se limitent à des raids lancés le plus souvent depuis la côte rifaine vers l'Espagne (voir ceux contre les villes de Bétique dans les années 170). Est-ce ce caractère très isolé de la piraterie qui explique que la surveillance des mers se faisait plutôt à partir des côtes? On possède ainsi l'attestation d'un chevalier anonyme, vers la fin du Ir siècle, dont la dernière milice était une préfecture d'aile en même temps que la surveillance de la côte maritime en Maurétanie césarienne (CIL, XI, 5744), d'un autre préfet chargé de la surveillance des côtes à la tête de soldats de la $14^{\text {ème }}$ cohorte urbaine, près d'Hippone (ILAlg. I, 30). Augustin parle également d'un tribun préposé aux côtes dans la même région, en 410, mais c'est dans le contexte troublé des vols d'enfants vendus comme esclaves (lettre 115 : misi ad tribunum, qui custodiendo littori constitutus est. Misit militares). En effet, il n'y a pas de flotte de guerre africaine, tout au plus une escadre à Césarée, attestée par la présence de quelques inscriptions qui semblent indiquer que l'escadre était détachée de 
la flotte d'Alexandrie plutôt que de celle de Misène. De Césarée, les marins pouvaient être envoyés pour des missions plus ou moins longues dans d'autres villes comme Saldae ou Portus Magnus (Saint Leu).

8 Les naufrages restaient donc le risque majeur, comme le montrent les épaves que l'archéologie sous-marine nous fait connaître. Son importance pour le renouvellement des perspectives d'étude de la navigation est considérable, mais malheureusement pour l'Afrique les données restent très rares. Cependant, des épaves avaient été retrouvées dès 1847 dans le port de Césarée (J. J. Bugnot et de Verneuil, « Esquisses historiques sur la Maurétanie césarienne et Iol Césarée », Rev. afr. 14, 1 870, p. 134-5) : «En 1847, en draguant le port, on rencontra les carcasses de deux galères romaines défendues par le sable et conservant leur forme antique. Quelles que furent les précautions que l'on apporta à leur enlèvement, les bois tombèrent en décomposition à mesure qu'on les retira. On ne put conserver que des masses de fonte servant de lest ». Les difficultés et la dispersion des recherches restées souvent confidentielles limitent les données récentes ; on peut néanmoins citer une épave du Bas Empire dans la baie du Lazaret à Hippone avec sa cargaison d'amphores africaines fabriquées à Nabeul et, pour le Maroc, les quelques remarques de $\mathrm{M}$. Ponsich au sujet des prospections dans la région du cap Spartel où la navigation est délicate. Elles aboutirent à la découverte de trois épaves antiques, démantelées par le ressac dans la baie de Tanger, avec des cols d'amphores des $\mathrm{II}^{\mathrm{e}}$ et $\mathrm{III}^{\mathrm{e}}$ siècles dans la première et d'époque augustéenne dans les deux autres; du cap Spartel, proviennent des pièces de plomb et un jas d'ancre et, au nord du ras Achakkar, en bordure du rivage, de nombreuses amphores furent retrouvées à marée basse.

9 L'intensité des relations maritimes ne fit que s'accroître au fur et à mesure que les provinces africaines, Proconsulaire et Numidie, jouaient un rôle de plus en plus important, par le volume des transactions autant que par leur diversité, avec Rome, du fait d'une prospérité économique de plus en plus affirmée avec des exportations agricoles (blé, huile), mais aussi celles de garum et de céramique africaine. Ces relations commerciales se développent aussi bien dans le cadre du ravitaillement officiel de Rome et des services de l'annone que du commerce libre ; le commerce du blé est très important: fourni dans le cadre de l'annone par les domaines impériaux et l'impôt, mais provenant aussi du marché libre, il est transporté à Ostie - et il y a jusqu'à trois rotations annuelles - par des propriétaires de bateaux, les naviculaires, avec lesquels l'État a passé un contrat. À Ostie, les naviculaires africains sont particulièrement nombreux; on les connaît par les mosaïques de la place des corporations où sont illustrés leurs ports d'origine, des pavements de pièces séparées qui constituaient les stationes de collèges d'armateurs ou de négociants d'une série de ports méditerranéens : pour l'Afrique, Carthage, Hippo Diarrythus, Curubis, Sabratha, Sullectum, mais aussi le petit port de Musluvium à côté de Saldae. Ces naviculaires pouvaient être à la tête d'affaires considérables, comme P. Aufidius Fortis qui concentre entre ses mains des fonctions non seulement de transporteur mais aussi de commerçant et qui contrôle toute une filière. Décurion d'Hippo Regius, il possède des domaines en Afrique proconsulaire, il est à la tête d'une entreprise très concentrée qui lui permet de contrôler le blé de sa production jusqu'à sa livraison au consommateur. Il est l'image de ces provinciaux, surtout africains à Ostie, qui contrôlent ainsi tout un secteur depuis la production jusqu'à la vente en passant par le transport. Les études de M. Cebeillac et M. Christol ont montré qu'un véritable lobby africain s'était constitué à Ostie au $\mathrm{II}^{\mathrm{e}}$ siècle et non sans conséquence sur les services de l'annone même, où l'on trouve souvent, aux 
postes de responsabilité, des chevaliers originaires de l'Afrique. Mais on possède aussi des témoignages beaucoup plus modestes sur les relations commerciales, comme ces deux poèmes de la région de Cirta mentionnant des marchands qui ont choisi de reposer dans leur terre natale (ILALg., II, 73, Rusicade: "il est natif de ce lieu, commerçant... Il a dû naviguer pour mener ses affaires » ; près de Sigus, ILALg., II, 6583 : "à force de naviguer et de voyager pendant bien des années, j'ai pu m'installer ici »). Outre le blé, l'huile, devenue produit annonaire à partir de Septime Sévère, vient de plus en plus souvent d'Afrique. Les grands flux suivent des routes commerciales pratiquées et connues depuis longtemps (voir Arnaud, 2005, p. 56). Mais il ne faut pas oublier non plus le rôle de la navigation dans les relations entre les différentes provinces, notamment entre la Tingitane et la Césarienne, comme le montre l'exemple de la mort de Lucceius Albinus, le procurateur des deux Maurétanies en 69, sur une plage, alors qu'il se rendait de Tingitane en Césarienne (Tacite, Histoires, II, 58); ni le rôle du cabotage, illustré par les nombreuses stations littorales de la voie maritime entre Tanger et Carthage dans l'Itinéraire Antonin. Le rôle du cabotage et des ports secondaires dans le commerce de redistribution apparaît à travers les vestiges d'installations, bien repérés par les prospections de P. Trousset le long du littoral tunisien, et par les amphores de la nécropole de Pupput qui proviennent aussi bien de Neapolis que d'Hadrumète, Leptiminus et Sullectum ; il est très concrètement illustré par une mosaïque de Sousse qui représente une scène de déchargement et de pesage d'une cargaison peut-être de lingots de plomb : le mât du navire est couché pour éviter l'effet du roulis sur le bateau mouillé près d'une plage; on est donc bien dans un contexte de redistribution par un cabotage local. La même intensité des activités de cabotage a été relevée par Ph. Leveau pour les régions littorales à l'est et à l'ouest de Césarée en relation avec l'exploitation des ressources des uillae et des carrières. On est beaucoup moins bien renseigné sur les conditions de la navigation fluviale pour la redistribution vers l'intérieur. On ne possède qu'un document, le tarif du péage du bac de Radès, sur le lac de Tunis, datant de la seconde moitié du V $V^{e}$ siècle (CIL, VIII, 24512).

10 L'intensité des relations avec Rome explique la multiplication des infrastructures, non seulement les ports dont le plus bel exemple de réalisation est fourni par Lepcis Magna sous l'impulsion de Septime Sévère, mais aussi la construction de vastes entrepôts (horrea) d'Etat, municipaux ou privés. On constate parfois que les ports changent de site, comme Choba : les premières traces d'occupation, à l'époque punique, se situent sur l'îlot de Mansourah; à l'époque romaine, le port se développe sur la côte à l'embouchure de l'oued Ziama et est abandonné, à la suite de son ensablement, à l'époque médiévale, pour se fixer à nouveau dans l'îlot de Mansourah. La part croissante des Africains dans la vie maritime est illustrée aussi par le grand nombre de mosaïques qui représentent des thèmes en relation avec la navigation, dont la plus célèbre est celle d'Althiburos où apparaissent schématiquement tous les types de navires avec leurs noms : voiliers de commerce (naues onerariae) aux formes bombées, à un seul mât et donc une voile, parfois surmontée d'une petite voile triangulaire, soit avec deux mâts dont l'un incliné vers l'avant à la proue ; galères effilées, plus rapides, moins nombreuses et moins utilisées pour le commerce, navires très spécialisés comme les hippagi qui avaient une porte sur le flanc permettant le transport des chevaux ou les lapidariae naves pour le transport des pierres, voire des obélisques, mais aussi toutes les barques et petites embarcations.

11 C'est par la mer que les Vandales, avec femmes et enfants, soit près de 80000 personnes, par une série de navettes sur de grosses barques et des navires de commerce 
réquisitionnés, passèrent en 429 du sud de l'Espagne vers la région de Tanger. Malgré l'absence de traditions maritimes, ils surent au mieux utiliser les potentialités que leur offrit la prise de Carthage en 439, avec son port et sa flotte. Si les Vandales n'ont pas constitué « un empire de la mer » sur le modèle de la Carthage punique (F. Gautier), ils disposaient d'une flotte qu'ils pouvaient développer grâce à l'arsenal de Carthage, aux ressources en bois des îles et, en hommes de l'Afrique (Sidoine Apollinaire, carmen $V$, 389-393). À la fin de la période vandale, Geilimer est encore en mesure d'envoyer en Sardaigne, pour mater une rébellion, 120 navires, «les meilleurs de sa flotte ", d'après Procope, (Bel. Vand., I, 11, 23). Les bateaux fournirent, au roi Geiseric (voir EB, XX, 1998, p. 3052-3055), dans son affrontement avec les Romains et les Byzantins, les moyens de protéger le territoire où il avait installé son peuple et de tirer parti de toutes les occasions de conquête (les Baléares, la Corse, la Sardaigne et temporairement la Sicile), avec notamment le raid de 455 qui débouche sur le pillage de Rome, la victoire obtenue par la ruse sur la flotte byzantine en 468 , et les opérations de pillage vers le sud de l'Italie et même jusque dans l'Adriatique. Cette insécurité en Méditerranée n'a pourtant pas interrompu les relations commerciales, comme le montre la présence de marchands orientaux à Carthage (Procope, I, 20, 4-6) et celle d'Africains à Byzance et en Italie.

Il est un passage d'un texte de l'Afrique tardive, la Passion de sainte Salsa de Tipasa, dans lequel l'auteur africain décrit parfaitement ce qu'est l'art de la navigation qui associe la nature, les bateaux et des hommes : il évoque les rythmes, les itinéraires et les dangers de la navigation avec la crainte essentielle des naufrages, y compris à proximité immédiate des côtes, mais il atteste aussi la place qu'occupe désormais la mer, non seulement dans son rôle économique mais aussi dans l'imaginaire des Africains, à la fin de l'antiquité : «Voici qu'un certain Saturninus arriva presque au même moment de la région des Gaules ; les flots étaient calmes, caressés de leurs brises sereines par les doux zéphyrs; près du port, il abattit les voiles et jeta l'ancre; il se fiait aux premiers moments de l'été et à ceux-là mêmes où, ainsi qu'on le dit d'après l'observation des signes, la mer est, comme à partir de ce jour, ouverte et où la houle déchaînée s'apaise, où les flots féroces, oubliant leur courroux, s'adoucissent et font, de tout rivage, un port, quand, même dans les nuits sans lune, les nefs fendent les flots et maintiennent leur cap sans danger; ainsi, sur son navire au mouillage, Saturninus jouissait d'un sentiment de parfaite sécurité. Mais soudain monte le grondement d'une mer devenue folle, les flots s'élancent jusqu'aux astres, et voilà que des rouleaux menaçants bouleversaient toute l'étendue salée. Les lames se lançaient à l'assaut les unes des autres [...] Un épais rideau de nuages avait caché le ciel [...] Dans un étincellement sinistre, avec des secousses épouvantables, passaient les feux d'éclairs incessants et, sous l'arc de la voûte céleste, dans un grondement assourdissant, vibrait le fracas du tonnerre. Les rivages étaient écrasés sous le choc brutal de montagnes liquides; leur reflux en tourbillonnant fait voler des nuages de sable et les gouttes d'écume qu'il projette en brume avoisinent le ciel. Tantôt s'élançant par la poupe, les flots couraient jusqu'à la proue, tantôt bondissant par la proue, la lame se jetait jusqu'à la poupe ; fouetté par toutes les bourrasques, tel un récif nu, le navire était emporté» (Traduction du GRAA, Montpellier).

13 La mer et la navigation en sont donc venues à jouer un rôle considérable dans la vie quotidienne des Africains; mais si les populations du littoral se tournèrent volontiers vers elle, celles de l'intérieur, au contraire, à l'instar de saint Augustin, qui choisit de se 
rendre par route plutôt que par mer d'Hippone à Césarée de Maurétanie, se montrèrent toujours méfiants vis-à-vis de l'univers marin.

\section{BIBLIOGRAPHIE}

ARNAUD P., 2005 - Les routes de la navigation antique. Itinéraires en Méditerranée, Paris.

BRIDOUX V., 2006 - Les royaumes d'Afrique du Nord de la fin de la deuxième guerre punique à la mort du roi Bocchus, thèse dactylographiée, Université de Paris I.

BEN MANSOUR S., 1998 - « La navigation d'après la mosaïque », Africa, 16, p. 15-30.

G. CAMPS, 1960 - Massinissa ou les débuts de l'histoire, Lybica, VIII.

J. DESANGES, 1978 - Recherches sur l'activité des Méditerranéens aux confins de l'Afrique, Rome.

M. JODIN, 1964 - « Contribution à l'atlas archéologique du Maroc : la région de Tanger », BAM, 5,

p. 262.

S. LANCEL, 1992 - Carthage, Fayard.

J.-P. LAPORTE, 2008 - «L'Algérie et la mer dans l'Antiquité : notes de lecture », dans J. NAPOLI éd., Ressources et activités maritimes des peuples de l'Antiquité, Actes du Colloque de l'Université du Littoral Côte d'Opale, (Boulogne-sur-mer, 12-14 mai 2005), Les Cahiers du Littoral, sér. 2, 6, Boulogne-sur-mer, p. 157-173.

LEVEAU Ph., 1984 - Caesarea de Maurétanie, Rome.

LUQUET J., 1973 - « Note sur la navigation de la côte atlantique du Maroc », BAM, 9, p. 297-306.

POMEY P., dir., 1992 - La navigation dans l'Antiquité, Arles.

VUILLEMOT G., 1965 - Reconnaissance aux échelles puniques d'Oranie, Autun.

\section{INDEX}

Mots-clés : Antiquité, Commerce, Economie, Navigation 